\title{
EQUAÇÕES NACIONAIS PARA A ESTIMATIVA DA GORDURA CORPORAL DE MILITARES DO EXÉRCITO BRASILEIRO
}

\author{
National equations for the estimate of the corporal fat of military \\ of the Brazilian Army
}

\author{
Ricardo Costa de Almeida Rêgo ${ }^{1}$, Marco Túlio Baptista ${ }^{1}$, Marcelo Salem ${ }^{2,3}$
}

\section{Resumo}

O homem sempre desejou possuir o conhecimento dos elementos constituintes do seu organismo (Rodriguez-Añez e Pires Neto, 1999). A estimativa e o cálculo da gordura corporal podem ser realizados por vários métodos laboratoriais, sendo diretos ou indiretos. Todavia, o único e mais preciso método direto para se quantificar a gordura corporal é através da dissecação cadavérica (Petroski, 2003). Apesar da existência de uma variedade de métodos indiretos, precisos e modernos, seus usos não são recomendados para avaliar um grande número de pessoas, já que utilizam equipamentos caros, gastam tempo considerável e necessitam de profissionais altamente qualificados (Norton e Olds, 1996). A busca de técnicas mais fáceis e bem mais econômicas fez com que vários profissionais, no Brasil e no exterior, procurassem uma solução mais prática e mais barata para os métodos antropométricos, que preconizam as medidas das dobras cutâneas, dos perímetros musculares e dos diâmetros ósseos, realizados fora dos laboratórios. Apesar da disponibilidade de centenas de equações, o uso destas não deve ser indiscriminado, pois, a não ser que sejam validadas para grupos de sujeitos com diferentes características, só devem ser utilizadas em grupos para os quais foram desenvolvidas e validadas (Salem, 2004). Embora os métodos laboratoriais para estimar a densidade corporal sejam considerados mais precisos, eles, freqüentemente, não são práticos para emprego clínico ou para testes de massa, como é o caso do Exército Brasileiro (EB). Portanto, este estudo objetivou divulgar os trabalhos nacionais que desenvolveram e/ou validaram equações para o fracionamento da composição corporal de militares do EB, através de equações específicas, para a estimativa da densidade e/ou gordura corporal, a partir de medidas antropométricas. Com o presente estudo, conclui-se que existem equações que estimam com acurácia e validade a composição corporal de militares do EB. Entretanto, não é possível apontar uma única equação para ser utilizada para toda a população do $E B$, face às diferenças da composição corporal entre os sexos, idades e características étnicas. Assim, com relativa atenção às características da amostra, podem ser utilizadas as seguintes equações para militares do EB: Salem (2006); Salem, Fernandes Filho e Pires Neto (2004); Rodriguez-Añez e Pires Neto (1999); Glaner e Rodriguez-Añez (1999); Souza, Pires Neto e Guimarães (1998); e Lopes e Pires Neto (1997).

Palavras-chave: Composição Corporal, Equações, Densidade Corporal, Gordura Corporal, Exército Brasileiro.

\begin{abstract}
The humanity always desire having the knowledge of the elements that composes your body (Rodriguez-Añez e Pires Neto, 1999). The estimation and the calculus of body fat can be realized through several laboratorial methods, directs or indirects. Nevertheless, the only and most precise direct method to quantify body fat is dissecting a cadaver (Petroski, 2003). In spite of having a variety of precise and modern indirect methods, they are not recommended to evaluate a great number of people, because it is necessary to use expensive equipments, a big amount of time is required, and highly-qualified professionals are needed
\end{abstract}

1. Escola de Educação Física do Exército - Rio de Janeiro - RJ - Brasil.

2. Instituto de Pesquisa da Capacitação Física do Exército - Rio de Janeiro - RJ - Brasil.

3. Fundação Oswaldo Cruz ( FIOCRUZ ) - Rio de Janeiro - RJ - Brasil.

Recebido em 11.07.2007. Aprovado em 10.12.2007.

Revista de Educação Física 2008;140:27-42 
(Norton e Olds, 1996). The searches for easier and more economic techniques made a lot of professionals, in Brazil and abroad, look for a practical and less expensive solution in the anthropometric methods, which commends the measurements of skin folds, muscular perimeters and bone diameters, realized outside the laboratories. Despite having hundreds of equations, the use of them cannot be indiscriminately, unless they are validated to a group of individuals with different characteristics, otherwise they can only be used for groups whom they were developed and validated (Salem, 2004). Although the laboratorial methods to estimate the corporal density be considerate most precise, they are not practical to be used in clinical employ or tests with a large population, like in the Brazilian Army. However, this study aimed publicize national works that developed and/or validated equations to the division of corporal composition of militaries from the Brazilian Army, through specific equations, to assessment of body density and/or body fat, with anthropometric measurement. With the present study, it is ended that there are equations what they appreciate with accuracy and validity to body composition of militaries of the Brazilian Army. Meantime, it is not possible to point to only one equation to be used for the whole population of the Brazilian Army, face to the differences between the body composition between sex, age and ethnic characteristics. So, with relative attention to the characteristics of the sample, the next equations can use for militaries of the Brazilian Army: Salem (2006); Salem, Fernandes Filho e Pires Neto (2004); Rodriguez-Añez e Pires Neto (1999); Glaner e Rodriguez-Añez (1999); Souza, Pires Neto e Guimarães (1998); e Lopes e Pires Neto (1997).

Key words: Corporal Composition, Equations, Body Density, Body Fat, Brazilian Army.

\section{INTRODUÇÃO}

Sempre foi um desejo do homem possuir o conhecimento dos elementos que constituem o seu organismo. Os motivos que o induzem a esta procura podem ser variados, como, por exemplo, razões estéticas ou de aparência externa do corpo, por interesse na promoção e na manutenção da saúde, ou pode ter um fim muito mais utilitarista, como a influência no trabalho, na prática dos desportos e no treinamento de militares e atletas (Rodriguez-Añez e Pires Neto, 1999).

O interesse pelo fracionamento e pelo cálculo dos componentes corporais surgiu na década de 40, quando o Dr. Albert Behnke, médico da Marinha dos Estados Unidos, considerado a maior autoridade em composição corporal, realizou um trabalho de medidas corporais, visando fracionar a composição corporal. Seu trabalho utilizou medidas de estatura, de forma e de estrutura de 25 jogadores profissionais de futebol americano. Neste estudo, foi observado que 11, dos 17 jogadores, considerados obesos pela tabela de peso e altura, utilizada na época como padrão de medida da composição corporal, possuíam a gordura corporal relativamente baixa e que este "excesso de peso", na realidade, era devido ao desenvolvimento da massa muscular (Katch e McArdle, 1996).

Com o pioneirismo de Behnke, vários pesquisadores passaram a ter interesse em fracionar a composição corporal, visando obter informações detalhadas e importantes sobre as dimensões do corpo humano, visto que o tipo corporal fornece muito mais informações do que simplesmente proporções corporais (Salem et al., 2007).

Autores como Heyward e Stolarczyk (2000), além de avaliarem a quantidade total e regional de gordura corporal para identificar riscos à saúde, afirmam que são várias as aplicações da composição corporal, entre elas: identificar riscos à saúde associados a níveis excessivamente altos e baixos de gordura corporal total; identificar riscos à saúde, associados ao acúmulo excessivo de gordura intra-abdominal; proporcionar entendimento sobre os riscos à saúde associados à falta ou ao excesso de gordura corporal; monitorar mudanças na composição corporal associadas a certas doenças; avaliar a eficiência de intervenções nutricionais e de exercícios físicos na alteração da composição corporal; estimar o peso corporal ideal de atletas e não atletas; formular recomendações dietéticas e prescrições de exercícios físicos; e monitorar mudanças na composição corporal, associadas ao crescimento, desenvolvimento, maturação e idade.

Com o intuito de obter informações mais detalhadas, diversos estudos foram realizados para fracionar a composição corporal em compartimentos. O modelo de divisão da Massa Corporal Total (MCT) mais comum é através de dois compartimentos: Massa Gorda (MG) e Massa Livre de Gordura (MLG). A MG caracteriza-se pela soma de todos os lipídios corporais e a MLG pela soma 
da água, das proteínas e dos componentes minerais do corpo (Heyward e Stolarczyk, 2000).

Vários métodos podem ser utilizados para estimar a $M G$, entre eles: os métodos laboratoriais, podendo ser diretos ou indiretos; e os métodos de campo, sendo estes indiretos. O único e mais preciso método direto para se quantificar a gordura corporal, entretanto, é a dissecação cadavérica (Petroski, 2003).

Hoje em dia, alguns métodos de laboratório, bastante sofisticados, são utilizados para estimar a gordura corporal (Norton e Olds, 1996). Dentre eles, pode-se citar: o da condutividade elétrica total do corpo (Malina, 1987), o ultrasônico (Katch, 1983) e o do scanner com raios infravermelhos (McLean e Skinner, 1992).

Outros métodos podem ser encontrados com a mesma finalidade: a absortometria radiológica de dupla energia (DEXA), a bioimpedância elétrica, a densitometria, a pletismografia, a hidrometria, a espectometria, a ultra-sonografia, a tomografia computadorizada, a ressonância magnética, a ativação de nêutrons, a interactância de raios infravermelhos, a antropometria, a excreção de creatinina, a creatinina sérica, a absorção fotônica, a radiografia e a 3-metil-histidina urinária (Pollock e Wilmore, 1993).

Como se observa, existe uma variedade de métodos bem precisos e modernos. Entretanto, não são recomendados para avaliar um grande número de pessoas, já que utilizam equipamentos caros, gastam um tempo considerável e necessitam de profissionais altamente qualificados (Norton e Olds, 1996). Além disso, em alguns desses métodos, o avaliado é exposto à radiação e substâncias tóxicas (Lukaski, 1987; Deurenberg, Westrate e Hautvast, 1989; Baumgarter, Chumlea e Roche, 1990).

Face ao exposto, técnicas mais simples e econômicas foram buscadas por diversos profissionais para estimar a gordura corporal. Uma solução encontrada foi o método antropométrico, por ser prático e menos dispendioso. Este método preconiza as medidas das dobras cutâneas (DC), dos perímetros musculares e dos diâmetros ósseos, realizados fora dos laboratórios (Petroski, 2003). Assim, o método antropométrico é caracterizado pelo uso destas variáveis, em equações, para estimar a densidade corporal e/ou percentual de gordura (\%G).
Desse modo, o cálculo da gordura corporal, a partir de medidas antropométricas, é realizado, desenvolvendo-se e validando-se equações de regressão para esse fim (Salem et al., 2007). Por isso, verifica-se que as informações antropométricas são valiosas no que se refere à predição e à estimativa dos vários componentes corporais, no crescimento, no desenvolvimento e no envelhecimento (Heyward e Stolarczyk, 2000).

Além disso, existem várias vantagens no uso da técnica antropométrica: a boa relação das medidas antropométricas com a densidade corporal obtida através dos métodos laboratoriais; o uso de equipamentos de baixo custo financeiro; a facilidade e a rapidez na coleta de dados; e a não invasividade do método (Petroski, 2003).

Sendo assim, observa-se que o método antropométrico tem se apresentado como um dos mais práticos, não-invasivo e que utiliza equipamentos de baixo custo, quando comparado com outros métodos, além de correlacionar-se, significativamente, com a densidade corporal obtida através da pesagem hidrostática (Lohman, 1992; Jackson e Pollock, 1982).

De acordo com Norton e Olds (1996), a maioria das equações de predição foi desenvolvida usando métodos de laboratório como a densimetria hidrostática, ou seja, a medição da Densidade Corporal (D) utilizando-se a pesagem hidrostática. Segundo Lohman (1992), muitos peritos consideram a medida da densidade corporal como o procedimento padrão para a avaliação da composição corporal.

$\mathrm{Na}$ literatura atual, verifica-se que a pesagem hidrostática $(\mathrm{PH})$ tem sido considerada como o método de laboratório não-invasivo mais aceito para os estudos da composição corporal e que, mesmo após todas as adaptações que o método já sofreu, é, ainda hoje, considerado o procedimento padrão em muitos laboratórios, com aplicação na atividade física, na nutrição e no controle de peso (Petroski e Pires Neto, 1992).

Em todo o mundo, várias equações têm sido desenvolvidas com o objetivo de quantificar a gordura corporal e, a partir daí, relacioná-la com diversas doenças e com o risco coronariano. Desse modo, a disponibilidade de equações para predição da composição corporal é diversificada (Salem et al., 2007). 
Contudo, apesar da existência de centenas de modelos matemáticos, o uso das equações não deve ser indiscriminado, pois, a não ser que sejam validadas para grupos de sujeitos com diferentes características, só devem ser utilizadas em grupos para os quais foram desenvolvidas e validadas (Petroski, 1995).

Além disso, na utilização de uma equação para estimar a gordura corporal, deve-se tomar grande cuidado na escolha do modelo apropriado, pois características, como idade, gênero e variáveis antropométricas, devem ser consideradas, já que o uso de um método indireto para estimar os componentes corporais pode gerar vários erros (Salem et al., 2007).

Com o exposto, podem ser citados dois tipos de equações de predição para fracionamento da composição corporal: as equações generalizadas e as específicas. As equações generalizadas são desenvolvidas utilizando grandes amostras heterogêneas de idade, de gordura corporal e de aptidão física. As específicas, são aquelas desenvolvidas a partir de populações homogêneas (Heyward e Stolarczyk, 2000).

Sabe-se que a principal vantagem de uma equação específica, quando aplicada à sua população de origem, é a acuracidade. Já não podemos dizer o mesmo quando uma equação específica é utilizada para sujeitos com características diferentes da população que a gerou (Heyward e Stolarczyk, 2000).

Ainda, conforme Salem (2007), existem muitas equações para predição da gordura corporal validadas para a população brasileira, porém, em estudos nacionais, permanece a utilização de equações estrangeiras. Isso se deve a diversos fatores, entre eles, o desconhecimento de equações nacionais.

Assim, verifica-se a necessidade de utilização de equações específicas para determinadas populações. Entre estas populações, pode-se fazer referência aos militares brasileiros.

A carreira militar exige de seus profissionais uma condição física mínima, suficiente para o desempenho de funções militares específicas, em tempos de paz e de guerra, fazendo com que todos que optaram pela carreira das armas precisem manter a saúde e a forma física constantemente.
A necessidade de uma preparação diferenciada, para suportar as exigências requeridas pela profissão, faz com que haja a necessidade do desenvolvimento e da validação de equações específicas para a avaliação do estado de saúde e da conseqüente operacionalidade da Força Terrestre.

Alguns estudos já foram realizados por autores nacionais e, por este motivo, este artigo objetiva divulgar os trabalhos nacionais que desenvolveram e/ou validaram equações para o fracionamento da composição corporal de militares do Exército Brasileiro, através de equações específicas, para a estimativa da densidade e/ou gordura corporal, a partir de medidas antropométricas.

\section{METODOLOGIA}

Foram levantados, na literatura brasileira, estudos que desenvolvessem ou validassem equações para predição da composição corporal para militares do Exército Brasileiro, conforme descrito a seguir.

\section{Souza, Pires Neto e Guimarães (1998)}

O trabalho realizado por estes pesquisadores visou a comparação e a validação cruzada de equações antropométricas e de impedância bioelétrica para estimar a massa corporal magra em militares.

Este estudo foi realizado no Núcleo de Preparação de Oficiais da Reserva (NPOR) de Artilharia do Regimento Mallet, situado em Santa Maria, RS, onde foram avaliados 18 jovens brancos, voluntários, do sexo masculino, com idades entre 18 e 19 anos.

O trabalho objetivou comparar e realizar a validação cruzada da predição da massa corporal magra (MCM), proveniente de equações antropométricas e da impedância biolétrica, utilizando a MCM obtida após a densidade corporal $\left(\mathrm{MCM}_{\mathrm{DEN}}\right)$ da amostra acima citada, como variável critério. As variáveis analisadas foram: massa corporal (MC), estatura (E), perímetro abdominal umbilical (pabu), dobra cutânea abdominal horizontal (dcabh) e densidade corporal pelo protocolo da pesagem hidrostática. $\mathrm{O}$ aparelho VALHALLA $1990 \mathrm{~B}$ gerou a $\mathrm{MCM}_{\mathrm{VALH}}$, a resistência e a reatância.

Na determinação da densidade corporal (D), foi utilizada a equação apresentada em Behnke e Wilmore (1974), sendo $\mathrm{D}(\mathrm{g} / \mathrm{ml})=\mathrm{MC} /[(\mathrm{MC}-\mathrm{PH}) /(\mathrm{Da}-(\mathrm{VR}+0,1)]$, onde: $\mathrm{MC}$ é a massa corporal em kg; $\mathrm{PH}$ é o peso hidrostático 
QUADRO 1

EQUAÇÕES ANTROPOMÉTRICAS E DA IMPEDÂNCIA BIOLETRÉTRICA ANALISADAS.

\begin{tabular}{lcc}
\hline \multicolumn{1}{c}{ Autor } & Equação & EPE (kg) \\
\hline WB1 (Wilmore e Behnke, 1969)* & $\mathrm{MCM}=10,260+0,7927 \times \mathrm{MC}-0,3676 \times$ dcabh & 2,97 \\
WB2 (Wilmore e Behnke, 1969)* & $\mathrm{MCM}=44,636+1,0817 \times \mathrm{MC}-0,7396 \times$ pabu & 2,81 \\
SLA (Slaughter e Lohman, 1980)* & $\mathrm{MCM}=0,719 \times \mathrm{E}-63,9$ & 6,3 \\
LOH (Lohman) & $\mathrm{MCM}=0,485 \times\left(\mathrm{E}^{2} / \mathrm{R}\right)+0,388 \times \mathrm{MC}+5,32$ & 2,9 \\
SEG (Segal et al., 1988) & $\mathrm{MCM}=0,00066360 \times \mathrm{E}^{2}-0,02117 \times \mathrm{R}+0,62854 \times$ & 2,4 \\
& $\mathrm{MC}-0,12380 \times \mathrm{I}+9,33285$ & \\
\hline
\end{tabular}

* Equações antropométricas; ** Equações da impedância bioelétrica. Fonte: Souza et al.,1998.

em kg; Da é a densidade da água; VR é o volume residual; e 0,1 , o volume de ar no trato intestinal.

A MCM, determinada a partir da densidade corporal $\left(M_{D E N}\right)$, foi obtida através da seguinte seqüência: calculou-se a gordura corporal relativa, $\% G=(495 / D)-$ 450 (Siri, 1961); transformou-se a \%G em massa de gordura (MG, kg) pela equação: $M G=M C \times(\% G / 100)$; e calculou-se a massa corporal magra (MCM, kg) pela fórmula $\mathrm{MCM}=\mathrm{MC}-\mathrm{MG}$.

O aparelho VALHALLA, modelo 1990B, foi utilizado para gerar a $\mathrm{MCM}_{\mathrm{VALH}}$, como também, os valores em Ohms $(\Omega)$ da resistência $(R)$ e da reatância $\left(X_{c}\right)$.

As equações utilizadas para predição da MCM são apresentadas no QUADRO 1, onde, para a equação de Lohman (1992) e Segal et al. (1988), utilizou-se, unicamente, o valor de $\mathrm{R}$ fornecido pelo equipamento, estatura, massa corporal e idade do avaliado.

Os dados coletados foram processados no SPSS/PC+ e constou da estatística descritiva, correlação de Pearson $(R)$, coeficiente de determinação $\left(R^{2}\right)$, regressão linear simples, erro padrão de estimativa (EPE) e teste $t$ de Student para amostras dependentes. Para a realização da validação cruzada, foi utilizada a $\mathrm{MCM}_{\mathrm{DEN}}$, como a variável critério. Como variáveis preditoras, foram utilizados os valores da MCM obtidos através das equações antropométricas propostas por Wilmore e Behnke (1969) e por Slaughter e Lohman (1980), das equações decorrentes da impedância bioelétrica desenvolvidas por Lohman (1992) e Segal et al. (1988), bem como pelo MCM obtido através do equipamento de impedância $\left(\mathrm{MCM}_{\mathrm{VALH}}\right)$. A validação cruzada foi realizada conforme os critérios sugeridos em Heyward e Stolarczyk (1996), ou seja: a correlação deve ser $\geq 0,80$; não deve haver diferença estatística significativa, $p>0,05$; e o EPE deve ser menor do que $3,5 \mathrm{~kg}$.

A TABELA 1 apresenta os valores descritivos das características físicas, da composição e da impedância corporal dos avaliados e a TABELA 2, os valores descritivos da MCM estimada e os critérios.

Observa-se que a equação antropométrica de WB2 superestimou a MCM em 0,08\%, equivalente a $0,05 \mathrm{~kg}$, que representa a menor diferença percentual observada. A equação da impedância corporal de $\mathrm{LOH}$ subestimou a MCM em 4,22\%, o que representa $2,64 \mathrm{~kg}$, sendo esta a maior diferença percentual encontrada. A MCM calculada pela equação antropométrica de SLA foi subestimada em $0,22 \%$ em relação a $\mathrm{MCM}_{\mathrm{DEN}}$, o equivalente a $0,14 \mathrm{~kg}$.

A TABELA 3 mostra a validação cruzada da massa corporal magra proveniente das equações antropométricas, equações de impedância bioelétrica e a gerada pelo aparelho VALHALLA 1990B em relação a $\mathrm{MCM}_{\text {DEN }}$.

Após este estudo e de acordo com as recomendações de Heyward e Stolarczyk (1996) para validação cruzada, a única equação que alcançou validação foi a WB2, sendo esta uma equação antropométrica proveniente de universitários americanos.

Em virtude da amostra desse estudo ser constituída por jovens fisicamente ativos, a validação da equação WB2 pode estar associada aos achados de Wilmore (1974), onde as alterações nos perímetros estão relacionadas aos incrementos da MCM. Entretanto, sugere-se considerar o tipo de atividade física, desenvolvida pelos avaliados, para o desenvolvimento e validação de equações preditivas da 
TABELA 1

VALORES DESCRITIVOS DAS VARIÁVEIS ANTROPOMÉTRICAS, DA COMPOSIÇÃO CORPORAL E DA IMPEDÂNCIA BIOLÉTRICA DOS ALUNOS DO NPOR.

\begin{tabular}{lccc}
\hline Variáveis & Média & SD & Min - Max \\
\hline Estatura (cm) & 175,8 & 5,3 & $170,6-190,8$ \\
Massa $(\mathrm{kg})$ & 69,6 & 7,5 & $57,10-84,0$ \\
dcabh $(\mathrm{mm})$ & 11,17 & 4,10 & $3,68-17,4$ \\
pabu $(\mathrm{cm})$ & 77,43 & 3,98 & $72,1-86,0$ \\
$\% G_{\text {DEN }}$ & 9,89 & 2,24 & $6,68-15,58$ \\
MCM & 62,65 & 5,80 & $51,79-73,40$ \\
$\mathrm{R} \Omega^{*}$ & 484,11 & 32,69 & $419-533$ \\
Xc $\Omega^{*}$ & 59,17 & 12,12 & $44-84$
\end{tabular}

${ }^{*} \mathrm{R}=$ resistência, $\Omega$ (Ohms); Xc = Reatância, $\Omega$ (Ohms).

Fonte: Souza et al.,1998.

TABELA 2

VALORES MÉDIOS E DIFERENÇA \% ENTRE A MCM ESTIMADA E CRITÉRIO (KG).

\begin{tabular}{|c|c|c|c|c|}
\hline Equação & Média (kg) & SD & Dif. \%* & Min - Max \\
\hline WB1 & 61,36 & 5,83 & $-2,06$ & $53,54-70,85$ \\
\hline WB2 & 62,70 & 7,33 & $+0,08$ & $54,50-72,37$ \\
\hline SLA & 62,51 & 3,84 & $-0,23$ & $58,76-73,29$ \\
\hline $\mathrm{LOH}$ & 60,01 & 5,47 & $-4,21$ & $51,38-70,55$ \\
\hline SEG & 61,08 & 6,13 & $-2,51$ & $51,10-72,29$ \\
\hline $\mathrm{MCM}_{\mathrm{VALH}}$ & 61,96 & 4,84 & $-1,11$ & $54,20-71,30$ \\
\hline $\mathrm{MCM}_{\mathrm{DEN}}$ & 62,65 & 5,80 & & $51,79-73,40$ \\
\hline
\end{tabular}

${ }^{*}$ Dif $(\%)=$ Diferença em \% entre as médias da MCM predita e a $\mathrm{MCM}_{\mathrm{DEN}}$ critério.

Fonte: Souza et al.,1998.

TABELA 3

VALIDAÇÃO DAS EQUAÇÕES PREDITAS DA MCM PELA MCM DEN $_{\text {. }}$

\begin{tabular}{|c|c|c|c|c|c|c|}
\hline Equação & $r$ & $\mathbf{R}^{2}$ & EPE & Inclinação & Dif. Média, kg & $\mathbf{t}$ \\
\hline WB1 & $0,975^{\star}$ & 0,950 & 1,33 & $0,97001^{*}$ & $-1,28$ & $4,19^{*}$ \\
\hline WB2 & $0,956^{*}$ & 0,913 & 1,75 & $0,75609^{*}$ & $+0,05$ & $-0,09$ \\
\hline SLA & $0,759^{\star}$ & 0,576 & 3,88 & $1,14654^{*}$ & $-0,14$ & 0,16 \\
\hline $\mathrm{LOH}$ & $0,978^{*}$ & 0,956 & 1,22 & $1,03685^{\star}$ & $-2,64$ & $9,31^{*}$ \\
\hline SEG & $0,986^{*}$ & 0,972 & 0,98 & $0,93278^{*}$ & $-1,57$ & $6,43^{*}$ \\
\hline $\mathrm{MCM}_{\mathrm{VALH}}$ & $0,984^{*}$ & 0,968 & 1,05 & $1,17980^{*}$ & $-0,69$ & $2,20^{* *}$ \\
\hline
\end{tabular}

${ }^{*} \mathrm{p}<0,01 ;{ }^{* \star} \mathrm{p}<0,05$; Inclinação = inclinação da reta de regressão; Dif. Média = diferença méda, em kg, entre médias da MCM predita e MCM critério. Fonte: Souza et al.,1998. 
MCM via impedância corporal. Dessa forma, as equações

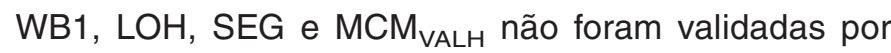
apresentarem diferenças significativas com a $\mathrm{MCM}_{\mathrm{DEN}}$, e a de SL, por elevado erro padrão de estimativa.

\section{Glaner e Rodriguez-Añez (1999)}

A pesquisa realizada por estes autores teve a intenção de validar procedimentos antropométricos para estimar a densidade corporal e o percentual de gordura em militares masculinos.

Foi realizada na $8^{\mathbf{a}}$ Circunscrição Militar, situada em Santa Maria, RS, onde foram coletados os dados de 50 militares, do sexo masculino, com idade média de 20,26 \pm 2,04 anos.

O objetivo da pesquisa foi verificar: a validade da equação de Katch e McArdle (1973), envolvendo os perímetros do braço, do antebraço e do abdômen, para estimar a densidade corporal; e o procedimento de Cohen (1986), envolvendo os perímetros do pescoço e de abdômen, para estimar o \% de gordura (\%G) para militares.

A amostra foi submetida aos seguintes procedimentos: mensuração da massa corporal e da estatura, com precisão de $100 \mathrm{~g} \mathrm{e} 0,1 \mathrm{~cm}$, respectivamente; e aferição dos perímetros corporais, conforme padronização de Katch e McArdle (1973) e Cohen (1986). Além destes, foi realizada a pesagem hidrostática, na posição grupada, conforme descrito em Petroski (1995). Assim, estimou-se o volume residual (R), pela equação de Goldman e Becklake (1959), que considera a idade e a estatura:

$$
V R=0,017 \times(\text { idade })+0,027 \times(\text { estatura })-3,477
$$

A densidade corporal mensurada (Dm) foi obtida pela equação:

$$
\begin{aligned}
\mathrm{Dm}(\mathrm{g} / \mathrm{ml}) & =\frac{\mathrm{MC}}{[(\mathrm{MC}-\mathrm{PS}) / \mathrm{Da}]-(\mathrm{VR}+0,1)} \\
\text { Onde: } & \\
\mathrm{MC} & =\text { Massa corporal }(\mathrm{kg}) . \\
\mathrm{PS} & =\text { Peso submerso na água }(\mathrm{kg}) . \\
\mathrm{Da} & =\text { Densidade da água } . \\
0,1 & =\text { Constante de gás gastrointestinal }(100 \mathrm{ml}) .
\end{aligned}
$$

O percentual de gordura $(\% G)$ estimou-se pela equação de Siri (1961):

$$
\% G=(495 / D)-450
$$

A massa de gordura (MG) e a massa corporal magra (MCM) foram obtidas pelas equações:

$$
\begin{aligned}
& M G=M C \times(100 / \% G) \\
& M C M=M C-M G
\end{aligned}
$$

Neste estudo, seguiu-se as sugestões de Lohman (1992) para a análise de validação, através dos cálculos do coeficiente de correlação linear de Pearson ( $r$ ), teste $t$ pareado $(t)$, erro constante $(E C)$, erro total $(E T)$ e erro padrão de estimativa (EPE). Para os cálculos estatísticos, utilizou-se o SPSS/PC.

Assim, pode-se verificar a TABELA 4, que caracteriza a amostra, e a TABELA 5, que aponta os resultados da validação da equação de Katch e McArdle (1973) e do procedimento de Cohen (1986).

Com as tabelas apresentadas, pode-se observar que os coeficientes de correlação entre as densidades mensurada e estimada, e entre o \%G mensurado e o estimado, apesar de serem altamente significativos, são de magnitude média. Evidenciou-se, através do teste $\mathrm{t}$ pareado, que o valor médio do $\% \mathrm{G}$, obtido pelo procedimento de Cohen (1986), não difere significativamente do valor médio do \%G mensurado, o que significa que este procedimento (Cohen) pode ser usado para estimar, com acuracidade, o \%G em militares masculinos entre 19 e 27 anos. O mesmo não acontece com a equação de Katch e McArdle (1973).

Com isso, conclui-se que o procedimento de Cohen atende a todos os critérios de validação sugeridos por Lohman (1992). Levando este fato em consideração e por este envolver somente dois perímetros corporais, não necessitando de cálculos, é uma extraordinária alternativa para estimar a quantidade de gordura relativa em militares brasileiros.

\section{Rodriguez-Añez e Pires Neto (1999)}

O trabalho destes autores foi realizado para desenvolver e validar equações de estimativa da densidade corporal de soldados e cabos do Exército Brasileiro (EB).

A coleta de dados antropométricos foi de uma amostra de 81 soldados e cabos do EB, com idades entre 18 e 22 anos, que cumpriam o serviço militar no Regimento Mallet, em Santa Maria, RS.

Foram mensuradas 13 dobras cutâneas (DC) e nove perímetros, além da massa corporal, da estatura e do peso hidrostático. 
TABELA 4

CARACTERÍSTICAS DESCRITIVAS DA AMOSTRA $(\mathrm{N}=50)$.

\begin{tabular}{|c|c|c|c|c|}
\hline Variáveis & $\mathbf{x}$ & $s$ & Mínimo & Máximo \\
\hline Idade (anos) & 20,26 & 2,04 & 19,00 & 27,00 \\
\hline Estatura (cm) & 173,06 & 6,56 & 157,00 & 187,50 \\
\hline Massa corporal (kg) & 68,36 & 8,34 & 51,40 & 98,60 \\
\hline $\begin{array}{l}\text { Densidade mensurada } \\
(\mathrm{g} / \mathrm{ml})\end{array}$ & 1,0699 & 0,0108 & 1,04 & 1,09 \\
\hline$\% G$ mensurada & 12,70 & 4,71 & 5,45 & 26,66 \\
\hline $\begin{array}{l}\text { Massa gorda mensurada } \\
(\mathrm{kg})\end{array}$ & 8,87 & 4,16 & 3,29 & 26,28 \\
\hline MCM mensurada $(\mathrm{kg})$ & 59,49 & 6,14 & 48,11 & 74,45 \\
\hline Perímetro do pescoço $(\mathrm{cm})$ & 36,62 & 1,85 & 33,50 & 44,00 \\
\hline $\begin{array}{l}\text { Perímetro do abdômen } \\
(\mathrm{cm})\end{array}$ & 79,29 & 6,25 & 69,00 & 104,00 \\
\hline $\begin{array}{l}\text { Perímetro do antebraço } \\
(\mathrm{cm})\end{array}$ & 26,69 & 1,60 & 25,00 & 31,50 \\
\hline Perímetro do braço $(\mathrm{cm})$ & 27,72 & 2,15 & 23,20 & 35,00 \\
\hline
\end{tabular}

Fonte: Glaner e Rodriguez-Añez, 1999.

TABELA 5

VALIDAÇÃO DA EQUAÇÃO DE KATCH E MCARDLE (K\&MCA) E DO PROCEDIMENTO DE COHEN.

\begin{tabular}{|c|c|c|c|c|c|c|}
\hline \multirow[t]{2}{*}{$x \mathrm{Dm}=1,0706 \pm 0,0100 \rightarrow(\mathrm{n}=47)$} & & \multicolumn{5}{|c|}{$x \% G$ derivado da $\mathrm{Dm}=12,38 \pm 4,35 \rightarrow(n=47)$} \\
\hline & $\mathbf{x}$ & $\mathbf{r}$ & $\mathbf{t}$ & EC & ET & EPE \\
\hline De: K\&McA (n=47) & $1,0752 \pm 0,0062$ & $0,5991^{1}$ & $3,72^{1}$ & $-0,0045$ & 0,0094 & 0,0080 \\
\hline$\% G: \operatorname{K} \& M c A(n=47)$ & $10,42 \pm 3,55$ & $0,5953^{1}$ & $-3,70^{1}$ & 1,96 & 4,09 & 3,50 \\
\hline \multicolumn{7}{|c|}{$x \% G$ derivado da $\mathrm{Dm}=12,70 \pm 4,71 \rightarrow(n=50)$} \\
\hline$\% G$ : Cohen $(n=50)$ & $13,74 \pm 5,03$ & $0,6802^{1}$ & $1,88^{2}$ & $-1,04$ & 4,00 & 3,45 \\
\hline
\end{tabular}

Para o desenvolvimento das equações, utilizou-se a regressão múltipla de Stepwise. Para a validação, determinou-se a média e o desvio padrão, a correlação de Pearson, o teste t pareado, o erro constante, o erro total (ET) e o erro padrão de estimativa (EPE). Foram propostas oito equações específicas para a predição da densidade corporal (TABELAS 6 e 7).

Pode-se conlcuir que, dentro das limitações inerentes a este estudo, as equações específicas propostas foram válidas para estimar a densidade corporal de soldados e cabos do EB que se enquadram, em valores antropométricos, entre os valores mínimos e máximos das variáveis dos grupos que geraram e validaram as respectivas equações.

\section{Salem, Fernandes Filho e Pires Neto (2004)}

Neste trabalho, buscou-se desenvolver e validar equações antropométricas específicas para a determinação da densidade corporal de mulheres militares do Exército Brasileiro (EB). 
TABELA 6

VALORES DESCRITIVOS DAS VARIÁVEIS BÁSICAS DAS AMOSTRAS DE REGRESSÃO E VALIDAÇÃO.

\begin{tabular}{|c|c|c|c|c|c|c|}
\hline \multirow[b]{2}{*}{ Variáveis } & \multicolumn{3}{|c|}{$\begin{array}{l}\text { Regressão } \\
(n=64)\end{array}$} & \multicolumn{3}{|c|}{$\begin{array}{c}\text { Validação } \\
(n=17)\end{array}$} \\
\hline & Média & $\mathbf{s}^{*}$ & Variação & Média & $\mathbf{s}$ & Variação \\
\hline Idade (anos) & 19,49 & 0,94 & $18,56-22,04$ & 18,68 & 0,06 & $18,54-18,77$ \\
\hline Peso (kg) & 65,50 & 7,82 & $51,4-88,6$ & 66,34 & 7,71 & $51,4-83,0$ \\
\hline Estatura $(\mathrm{cm})$ & 172,28 & 7,17 & $157,0-187,5$ & 169,12 & 7,72 & $157,0-187,0$ \\
\hline Densidade (g/ml) & 1,0693 & 0,009 & $1,0871-1,0494$ & 1,0652 & 0,010 & $1,0854-1,0494$ \\
\hline$\% G$ & 12,97 & 4,08 & $5,36-21,69$ & 14,73 & 4,44 & $6,42-21,69$ \\
\hline$M G(k g)$ & 9,00 & 3,33 & $3,27-17,40$ & 9,92 & 3,59 & $3,30-16,97$ \\
\hline MCM (kg) & 59,49 & 6,22 & $48,10-74,55$ & 56,41 & 5,79 & $48,10-66,03$ \\
\hline
\end{tabular}

Fonte: Rodriguez-Añez e Pires Neto, 1999.

TABELA 7

EQUAÇÕES ESPECÍFICAS PARA A ESTIMATIVA DA DENSIDADE CORPORAL DE SOLDADOS E CABOS DO EB.

\begin{tabular}{|c|c|c|c|}
\hline Equação & $\mathbf{R}$ & $\mathbf{R}^{2}$ & EPE \\
\hline $\begin{array}{l}\text { DC }=1,12227-0,00249263(X 3)+0,0004989252(X 2)^{2}+0,001926203(P P E S) \\
-0,0008869007(P A B D)-0,000523489(P C X S)\end{array}$ & 0,868 & 0,749 & 0,0045 \\
\hline $\begin{array}{l}\text { DC }=1,12432-0,00170410(\mathrm{X} 3)+0,00002110497(\mathrm{X} 3)^{2}+0,001996741(\mathrm{PPES}) \\
-0,000928645(\mathrm{PABD})-0,000466887(\mathrm{PCXS})\end{array}$ & 0,874 & 0,765 & 0,0043 \\
\hline $\begin{array}{l}\text { DC }=1,14293-0,000175058(X 6)+0,00001569687(X 6)^{2}+0,002012604(\text { PPES) } \\
-0,00101592(\text { PABD })-0,000426929(P C X S)\end{array}$ & 0,889 & 0,790 & 0,0041 \\
\hline $\begin{array}{l}\text { DC }=1,14981-0,00159294(X 7)+0,00001168942(X 7)^{2}+0,002025870(\text { PPES }) \\
-0,00104841(\text { PABD })-0,000414721(\mathrm{PCXS})\end{array}$ & 0,892 & 0,797 & 0,0040 \\
\hline $\begin{array}{l}\text { DC }=1,15101-0,00144732(\mathrm{X} 10)+0,000009398513(\mathrm{X} 10)^{2}+0,002136568(\mathrm{PPES}) \\
-0,00106070(\mathrm{PABD})-0,000470059(\mathrm{PCXS})\end{array}$ & 0,897 & 0,805 & 0,0039 \\
\hline $\begin{array}{l}\text { DC }=1,14673-0,00124366(\mathrm{X} 11)+0,000006973939(\mathrm{X} 11)^{2}+0,002167078(\mathrm{PPES}) \\
-0,001103163(\mathrm{PABD})-0,000461764(\mathrm{PCXS})\end{array}$ & 0,896 & 0,804 & 0,0039 \\
\hline $\begin{array}{l}\text { DC }=1,14908-0,00117048(X 14)+0,000005842855(X 14)^{2}+0,00224425(\text { PPES) } \\
-0,00102242(P A B D)-0,000485424(P C X S)\end{array}$ & 0,898 & 0,806 & 0,0039 \\
\hline $\begin{array}{l}\text { DC }=1.15141-0,00112681(\mathrm{X} 16)+0,000005325475(\mathrm{X} 16)^{2}-0,002183359(\mathrm{PPES}) \\
-0,00103708(\mathrm{PABD})-0,000474348(\mathrm{PCXS})\end{array}$ & 0,896 & 0,804 & 0,0039 \\
\hline $\begin{array}{l}\text { DC }=1,15592-0,001105988(X 17)+0,000004468730(X 17)^{2}+0,002170514(P P E S) \\
-0,00105844(\text { PABD })-0,000458337(P C X S)\end{array}$ & 0,862 & 0,796 & 0,0046 \\
\hline
\end{tabular}

\footnotetext{
Onde: X2 = dobra cutânea (dc) abdominal horizontal;

$\mathrm{X} 3=\Sigma$ dc abdominal vertical (ABDV) + dc suprailíaca oblíqua (SIO); $\mathrm{X} 6=\Sigma \mathrm{dc}$ adov $+\mathrm{SIO}+$ coxa medial (CXM);

$\mathrm{X} 7=\Sigma \mathrm{DC} A \mathrm{ABDV}+\mathrm{SIO}+\mathrm{CXM}+\mathrm{TR}$ (dc triciptal);

$\mathrm{X} 10=\Sigma \mathrm{DC} A \mathrm{ABDV}+\mathrm{SIO}+\mathrm{CXM}+\mathrm{TR}+\mathrm{PT}($ dc peitoral);
}

$\mathrm{X} 14=\Sigma \mathrm{DC} A \mathrm{ABV}+\mathrm{SIO}+\mathrm{CXM}+\mathrm{TR}+\mathrm{PT}+\mathrm{AXO}$ (dc axilar oblíqua) + PAM (dc panturrilha medial); $\mathrm{X} 16=\Sigma \mathrm{DC} A \mathrm{ABD}+\mathrm{SIO}+\mathrm{CXM}+\mathrm{TR}+\mathrm{PT}+\mathrm{AXO}+\mathrm{PAM}+\mathrm{BI}$ (diâmetro biestiloidal);

$\mathrm{X} 17=\mathrm{X} 16=\Sigma \mathrm{DC} A \mathrm{ABDV}+\mathrm{SIO}+\mathrm{CXM}+\mathrm{TR}+\mathrm{PT}+\mathrm{AXO}+\mathrm{PAM}+\mathrm{BI}+\mathrm{SE}$ (dobra subescapular); PPES = perímetro do pescoço; PABD = perímetro abdominal e PCXS = perímetro da coxa superior. Fonte: Rodriguez-Añez e Pires Neto, 1999. 
TABELA 8

EQUAÇÕES ESPECÍFICAS PARA A ESTIMATIVA DA DENSIDADE CORPORAL DE MULHERES DO EB

\begin{tabular}{|c|c|c|}
\hline Equação & $\mathbf{R}$ & EPE \\
\hline $\mathbf{D}=1,0-0,000748(\mathrm{BIC}+\mathrm{TRI})+0,002538($ ANTE $)+0,0007667($ TORAX $)-0,00000995(\mathrm{CINT})^{2}$ & 0,798 & 0,00542 \\
\hline $\mathbf{D}=1,058-0,000763(\mathrm{BIC}+\mathrm{TRI})+0,002948(\mathrm{ANTE})-0,000836(\mathrm{CINT})$ & 0,780 & 0,00593 \\
\hline $\begin{array}{l}\mathbf{D}=1,022-0,000676(\mathrm{BIC}+\mathrm{TRI})+0,000005533(\mathrm{TORAX})^{2}-0,0000104(\mathrm{CINT})^{2}+0,00004012 \\
(\mathrm{ANTE})^{2}+0,008641(\mathrm{DBI})\end{array}$ & 0,822 & 0,00516 \\
\hline $\mathbf{D}=1,03-0,0007(\mathrm{BIC}+\mathrm{TRI})-0,00000603(\mathrm{CINT})^{2}+0,00005083(\mathrm{ANTE})^{2}+0,007819(\mathrm{DBI})$ & 0,802 & 0,00537 \\
\hline $\mathbf{D}=1,045+0,002079(\mathrm{PESC})-0,00112(\mathrm{CINT})-0,000736(\mathrm{PCOXA})+0,01142(\mathrm{DBI})$ & 0,710 & 0,00633 \\
\hline $\mathbf{D}=1,058+0,002142(\mathrm{PESC})+0,00004764(\text { ANTE })^{2}-0,0011(\mathrm{PCOXA})-0,00000885(\mathrm{CINT})^{2}$ & 0,689 & 0,00652 \\
\hline $\mathrm{D}=1,040-0,000611(\mathrm{BIC}+\mathrm{TRI})-0,000269(\mathrm{CINT}+\mathrm{PABDO})+0,01303(\mathrm{DBI})$ & 0,784 & 0,00555 \\
\hline $\mathbf{D}=1,095-0,000676(\mathrm{BIC}+\mathrm{TRI})-0,000198(\mathrm{CINT}+\mathrm{PABDO})$ & 0,720 & 0,00616 \\
\hline $\mathbf{D}=1,069-0,000796(\mathrm{BIC}+\mathrm{TRI})$ & 0,681 & 0,00645 \\
\hline D $=1,081-0,000649(\mathrm{BIC}+\mathrm{TRI})-0,000000380(\mathrm{BIC}+\mathrm{TRI})^{2}-0,00000326(\mathrm{CINT})^{2}$ & 0,711 & 0,00628 \\
\hline
\end{tabular}

Onde: $\mathbf{B I C}=$ dobra do bíceps; TRI = dobra do tríceps; $\mathbf{A N T E}=$ perímetro do antebraço; $\mathbf{T O R A X}=$ perímetro do tórax; $\mathbf{C I N T}=$ perímetro da cintura; $\mathbf{D B I}=$ diâmetro biestiloidal; PESC = perímetro do pescoço; PCOXA = perímetro da coxa; PABDO = perímetro abdominal; $\mathbf{R}=$ coeficiente de correlação múltipla e EPE = erro padrão da estimativa. Fonte: Salem et al., 2004.

A amostra foi constituída por 100 mulheres militares do $\mathrm{EB}$, independente de posto ou graduação, com idade entre 18 e 45 anos, da cidade do Rio de Janeiro, RJ.

Foram realizadas as medidas de 10 dobras cutâneas, de 10 perímetros, de três diâmetros, da massa corporal (MC), da estatura e da densidade corporal (D) através da pesagem hidrostática.

Para o desenvolvimento das equações (TABELA 8), como em outros estudos, foi realizada a análise de regressão Stepwise e, para sua validação, foram realizados os cálculos do coeficiente de correlação linear de Pearson ( $p \leq 0,05)$, teste $t$ de Student para comparação entre medidas $(p \leq 0,05)$, cálculo do erro constante $(E C)$, cálculo do erro técnico (ET) e erro padrão da estimativa (EPE).

Após a realização deste estudo, chegou-se à conclusão que foi possível desenvolver e validar equações específicas para a determinação da densidade corporal das mulheres militares do EB, servindo no Rio de Janeiro, a partir de 
TABELA 9

EQUAÇÕES ESPECÍFICAS PARA A ESTIMATIVA DA DENSIDADE CORPORAL DE ALUNOS DA ESEFEX.

\begin{tabular}{lcc}
\hline Equação & $\mathbf{R}$ & EPE \\
\hline $\mathbf{D C}=1,146-0,001(\mathrm{PABD})+0,001(\mathrm{MCT})-0,002(\mathrm{DTRI})$ & 0,830 & $0,0058\left(\mathrm{~g} / \mathrm{cm}^{2}\right)$ \\
$\% \mathbf{G}=0,497(\mathrm{PABD})-0,230(\mathrm{MCT})+0,793(\mathrm{DTRI})-20,504$ & 0,832 & 2,91 \\
\hline
\end{tabular}

Fonte: Salem, 2006.

variáveis antropométricas (dobras cutâneas, perímetros e diâmetros). Ocorreu uma correlação significativa ( $p \leq 0,05)$ entre a $D$, medida através da pesagem hidrostática, e a estimada pelas equações desenvolvidas.

Desse modo, as equações, apresentadas no trabalho em pauta, são válidas para a estimativa da $D$ de mulheres militares do EB que se encontram dentro dos limites de dois desvios padrões nas variáveis relatadas e com idade entre 18 e 45 anos.

\section{Salem (2006)}

A pesquisa realizada por este autor objetivou desenvolver e validar equações para a estimativa da porcentagem de gordura dos alunos do curso de instrutor da Escola de Educação Física do Exército (EsEFEx).

Participaram do estudo 20 militares, oficiais do Exército, alunos da EsEFEx do ano de 2006. Foram realizadas as medidas antropométricas de dobras e de perímetros, a pesagem hidrostática para medição da densidade corporal, além do levantamento da idade, da estatura e da massa corporal total (MCT).

Os testes estatísticos foram realizados através do pacote SPSS 10.0. A tentativa de validação foi realizada com as equações de Guedes (1985), com uma dobra cutânea, e Petroski (1996), com duas dobras cutâneas e com duas dobras e duas circunferências. A escolha destas três equações baseou-se no critério da simplicidade, principalmente pelo fato deste trabalho ser uma iniciação à prática científica. Para a validação das equações, foram utilizados os mesmos procedimentos empregados em outros estudos. O único diferencial foi o método de validação não seguir as recomendações de Lohman (1992) e, sim, a análise diagnóstica dos resíduos de regressão (TABELA 9).
Com este estudo, conclui-se que as fórmulas apresentadas possuem validade e devem ser utilizadas nesta população para estimar a densidade corporal.

\section{Lopes e Pires Neto (1997)}

Estes autores não desenvolveram uma equação, realizaram, porém, um estudo sobre a composição corporal em militares utilizando a densimetria, a antropometria e a impedância bioelétrica, através da comparação entre estes métodos.

O estudo foi realizado com uma amostra de 18 militares do sexo masculino, com idade média 18,67 \pm 0,48 anos, servindo na cidade de Santa Maria, RS. Esta amostra realizava atividade física cinco vezes por semana.

O protocolo de mensuração antropométrica deste estudo foi realizado através da determinação da massa e da estatura corporal, das dobras cutâneas (DC) _ subescapular (SE); triciptal (TR); biciptal (BI); peitoral (PT); axilar vertical (AXV); abdominal vertical (ABDV); suprailíaca vertical e oblíqua (SIV e SIO); coxa medial (CX); e panturrilha medial (PAN) _ conforme os procedimentos do Laboratório de Cineantropometria da Universidade Federal de Santa Maria e de Harrison et al. (1991).

A pesagem hidrostática foi realizada conforme os procedimentos descritos por Petroski e Pires Neto (1992).

Para comparar a densidade corporal (D), obtida através da pesagem hidrostática, utilizou-se oito equações preditas da densidade corporal, desenvolvidas por oito autores diferentes, que utilizam 2, 3 e 4 dobras cutâneas. Procurou-se comparar os resultados com equações generalizadas $(1,2,5)$, específicas para universitários e adultos jovens $(4,6,8)$ e específicas para atletas $(3,7)$, conforme a TABELA 10. 
TABELA 10

EQUAÇÕES PREDITAS DA DENSIDADE CORPORAL.

N Equações estimativas da densidade corporal (D) Idade

$1 \quad$ PETROSKI, 1995; PET (95)

$18-66$

$D C=1,10726863-0,00081201^{*}(x 1)+0,00000212^{*}(x 1)^{2}-0,00041761^{*}(I)$

2 DURNIN \& WOMERSLEY, 1974; D\&W (74)

$17-19$

$D C=1,1620-0,0630$ * $\operatorname{Lg} 10(x 2)$

$3 \quad$ THORLAND et al, 1984; THO (84)

14-19

$\mathrm{DC}=1,1136-0,00154^{*}(\mathrm{x} 3)+0,00000516^{*}(\mathrm{x} 3)^{2}$

$4 \quad$ GUEDES, 1985; GUE (85)

$18-28$

$\mathrm{DC}=1,17136-0,06706^{*} \operatorname{Lg} 10(\mathrm{x} 4)$

$5 \quad$ JACKSON \& POLLOCK, 1978; J\&P (78)

$18-61$

$\mathrm{DC}=1,1093800-0,0008267^{*}(\mathrm{x} 5)+0,0000016(x 5)^{2}-0,0002574^{*}(\mathrm{I})$

SLOAN, 1967; SLO (67)
DC $=1,1043-0,001327$ * (CX) $-0,001310 *($ SE $)$

$7 \quad$ FORSYTH \& SINNING, 1973; F\&S (73)

$19-22$

$D C=1,10300-0,00168$ * $(S E)-0,00127^{*}(A B D V)$

8

KATCH \& MCARDLE, 1973; K\&M (73)

$18-24$

$\mathrm{DC}=1,09665-0,00103^{\star}(\mathrm{TR})-0,00056^{\star}(\mathrm{SE})-0,00054^{\star}(\mathrm{ABDV})$

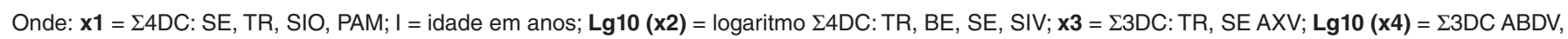
TR, SIO; $\mathbf{x} 5=\Sigma$ S3DC: CX, PT, ABDV.

NA = valor não apresentado

Fonte: Lopes e Pires Neto, 1997.

A densimetria foi realizada através da pesagem hidrostática, utilizando-se, como critério para a densidade corporal (D), a gordura corporal (\%G) e a massa corporal magra (MCLG). A D foi determina conforme a equação:

$$
D(g / m l)=\frac{P}{[(P-P a) / D a]-(V R+0,100)}
$$

Onde:

$$
\begin{aligned}
& \mathrm{D}=\text { Densidade corporal } \\
& \mathrm{P}=\text { Massa corporal }(\mathrm{kg}) \\
& \mathrm{Pa}=\text { Peso submerso na água }(\mathrm{kg}) \\
& \mathrm{Da}=\text { Densidade da água } \\
& \mathrm{VR}=\text { Volume residual }(\mathrm{L}) \\
& 0,1=\text { Constante de gás gastrointestinal }(100 \mathrm{ml}) \\
& \mathrm{VR}=0,017 \times \text { (idade })+0,027 \times(\text { estatura })-3,477
\end{aligned}
$$

O percentual de gordura ( $\% \mathrm{G})$ foi estimado pela equação de Siri (1961). A massa de gordura (MG) e a massa corporal magra (MCM) foram obtidas pelas equações:

$$
\begin{aligned}
& M G=M C \times(100 / \% G) \\
& M C M=M C-M G
\end{aligned}
$$

O método da impedância bioelétrica foi executado através da utilização dos aparelhos VALHALLA, modelo 1990B, e BIODYNAMICS, modelo 310 (1995).

Após a coleta de todos os dados, realizou-se a análise estatística, utilizando-se a estatística descritiva básica, coeficiente de relação de Pearson (r), teste t de Student dependente, para verificar se haviam diferenças estatisticamente significativas, $p<0,01$, entre as médias da densidade corporal, da gordura relativa, da gordura absoluta e da massa corporal livre de gordura, obtidas através da pesagem hidrostática, cada uma das equações 
TABELA11

CORRELAÇÃO E COMPARAÇÃO ENTRE OS RESULTADOS DA PESAGEM HIDROSTÁTICA, EQUAÇÕES ANTROPOMÉTRICAS PREDITIVAS DA DENSIDADE CORPORAL E IMPEDÂNCIA BIOELÉTRICA.

\begin{tabular}{|c|c|c|c|c|c|}
\hline Variáveis & & $D(g / c c)$ & $r$ & $t$ & $p$ \\
\hline \multirow[t]{2}{*}{$\mathrm{PH}$} & $x$ & 1,0764 & & & \\
\hline & $\mathrm{s}$ & 0,005 & & & \\
\hline \multirow[t]{2}{*}{1 - PET (95) } & $x$ & $1,0720^{\star}$ & 0,70 & $-4,43$ & 0,001 \\
\hline & $\mathrm{s}$ & 0,005 & & & \\
\hline \multirow[t]{2}{*}{2 - D\&W (74) } & $x$ & $1,0674^{\star}$ & 0,62 & $-7,73$ & 0,001 \\
\hline & $\mathrm{s}$ & 0,006 & & & \\
\hline \multirow[t]{2}{*}{$3-\mathrm{THO}(84)$} & $x$ & 1,0784 & 0,59 & 1,70 & 0,109 \\
\hline & $\mathrm{s}$ & 0,006 & & & \\
\hline \multirow[t]{2}{*}{4 - GUE (85) } & $x$ & 1,0740 & 0,64 & $-1,57$ & 0,135 \\
\hline & s & 0,009 & & & \\
\hline \multirow[t]{2}{*}{5 - J\&P (78) } & $x$ & $1,0828^{\star}$ & 0,78 & 8,01 & 0,001 \\
\hline & $\mathrm{s}$ & 0,005 & & & \\
\hline \multirow[t]{2}{*}{6 - SLO (67) } & $x$ & 1,0750 & 0,79 & $-1,59$ & 0,130 \\
\hline & $\mathrm{s}$ & 0,006 & & & \\
\hline \multirow[t]{2}{*}{7 - F\&S (73) } & $x$ & 1,0742 & 0,61 & $-1,87$ & 0,078 \\
\hline & $\mathrm{s}$ & 0,006 & & & \\
\hline \multirow[t]{2}{*}{$8-\mathrm{K} \& M(73)$} & $x$ & 1,0769 & 0,63 & 0,56 & 0,580 \\
\hline & s & 0,004 & & & \\
\hline \multirow[t]{2}{*}{$9-\mathrm{BIO}(95)$} & $x$ & $1,0728^{\star}$ & 0,83 & $-4,23$ & 0,001 \\
\hline & $\mathrm{s}$ & 0,005 & & & \\
\hline \multirow[t]{2}{*}{$10-$ VAL (90B) } & $x$ & 1,0747 & 0,79 & $-1,51$ & 0,149 \\
\hline & $\mathrm{s}$ & 0,006 & & & \\
\hline
\end{tabular}

Obs: * médias significativamente diferentes da média da PH pelo teste $\mathrm{t}(\mathrm{p}<0,01)$; BIO (95) BIODYNAMICS, modelo 310; VL (90B) = VALHALLA, modelo 1990B; valores t, r, p entre percentual de gordura decorrente da PH e percentual de gordura das demais variáveis; a densidade corporal para a Impedância Bioelétrica foi obtida substituindo o percentual de gordura da AIB na equação de Siri (1961). 
de regressão e análise da impedância bioelétrica. Utilizou-se o pacote estatístico SPSS/PC+ (1989).

A TABELA 11 aponta os resultados médios da comparação da pesagem hidrostática para a densidade corporal (D), gordura corporal relativa (\%G), massa de gordura (MG), massa corporal livre de gordura (MCLG), com as equações antropométricas preditivas da densidade corporal e análise de impedância bioelétrica, bem como o coeficiente de correlação.

Com o exposto, chega-se à conclusão de que as equações específicas para universitários, adultos e atletas jovens, predizem com maior acuracidade a densidade corporal dos militares da amostra do que as equações generalizadas, que não foram boas preditoras da $D$ e, conseqüentemente, do \%G, MG e da MCLG (Lopes e Pires Neto, 1997).

Além disso, uma limitação da AIB é o tipo de aparelho utilizado, pois este pode ser uma substancial fonte de erro. O BIODYNAMICS, modelo 310, superestimou o \%G e subestimou a MCLG em relação ao método de referência, enquanto que o VALHALLA, modelo 1990B, não apresentou diferença estatística significativa na gordura corporal relativa, mas subestimou a MCLG. Entretanto, a impedância bioelétrica apresentou maior correlação com a PH do que o método antropométrico (Lopes e Pires Neto, 1997).

\section{CONCLUSÃO}

Analisando os dados encontrados na literatura, diante da importância da composição corporal sobre os aspectos da saúde dos indivíduos, é necessário que os profissionais de saúde disponham de métodos confiáveis e de fácil utilização para sua validação. Conhecer a validade desses métodos é imprescindível para a aplicação dos mesmos, objetivando obter estimativas mais precisas da gordura corporal (Rezende et al., 2005).

Ressalta-se que, no Brasil, conforme Salem (2007) apresenta em seu estudo, já existem trabalhos que desenvolveram e validaram equações nacionais. Apesar de bastante utilizadas, as equações internacionais só podem ser utilizadas na população brasileira se forem validadas.

Sendo assim, os estudos listados nesta revisão são direcionados a uma população específica: de militares do Exército Brasileiro.

Estudos com militares brasileiros são facilitados: devido à voluntariedade das amostras; em função do grande efetivo existente; porque os pesquisadores recebem apoio freqüente dos comandantes das Organizações Militares; pelo fato dos militares realizarem exames médicos constantes e, consequentemente, sendo saudáveis; e pela possibilidade de utilização de amostras de várias regiões do país.

Os militares do Exército Brasileiro são um extrato da população brasileira. Assim, para brasileiros civis, enquadrados nos parâmetros antropométricos do grupo para o qual foi desenvolvida a equação, pode-se usar as equações com uma pequena margem de erro.

Face ao exposto, observa-se que existem equações que podem ser aplicadas aos militares do Exército Brasileiro com acurácia e validade. Contudo, não é possível determinar uma única equação para a população em questão, devido às variações existentes dentro da própria amostra: diferenças quanto à composição corporal entre os sexos; elevada quantidade de massa magra em homens e maiores percentuais de gordura em mulheres (Bembem et al., 1995); diferença de idade, o que acarreta alteração na mineralização óssea e hidratação da massa magra, redistribuição e aumento da gordura corporal (Martins et al., 2001); diferença entre os níveis de aptidão física; e características étnicas.

Sendo assim, é possível afirmar que a população referida, com relativa atenção às características da amostra, possui equações específicas e generalizadas para a estimativa da gordura corporal, através da comparação com outros métodos de predição da composição corporal.

Ainda, chega-se à conclusão que, apesar de equações nacionais terem sido publicadas e estarem disponíveis na internet, infelizmente, muitos pesquisadores brasileiros utilizam equações internacionais.

\section{REFERÊNCIAS BIBLIOGRÁFICAS}

BEMBEN MG, MASSEY BH, BEMBEN DA, BOILEAU RA, MISNER JE. Age related patterns in body composition for men aged 20-79 yr. Med Sci Sports Exerc 1995; 27(2):264-9. 
DURNIN JVGA, RAHAMAN MM. The assessment of the amount of the fat in the human body from measurements of skinfold thickness. British Journal of Nutrition 1967; 21:681-9.

ESTADO MAIOR DO EXÉRCITO. Manual de Treinamento Físico Militar (C 20-20). Brasília: EGGCF, 2002.

FERNANDES FILHO J. A prática da avaliação física. 2ª ed. Rio de Janeiro: Shape, 2003.

GLANER MF, RODRIGUEZ-AÑEZ CR. Validação de procedimentos antropométricos para estimar a densidade corporal e percentual de gordura em militares masculinos. Revista Brasileira de Cineantropometria \& Desempenho Humano 1999; $1(1): 24-9$.

GLANER MF, RODRIGUEZ-AÑEZ CR. Validação de equações para estimar a densidade corporal e/ou percentual de gordura para militares masculinos. Treinamento Desportivo 1999; 1(4):29-36.

HEYWARD VH, STOLARCZYK LM. Avaliação da composição corporal aplicada. São Paulo: Manole, 2000.

JACKSON AS, POLLOCK ML. Steps toward the development of generalized equations for prediction body composition of adults. Canadian Journal of Applied Sport Sciences 1982; 3(7):189-196.

KEYS A, BROZEK J. Body fat in adult man. Physiological Review 1953; 3(33):245-325.

LOHMAN TG. Advances in body composition assessment. Champaign: Human Kinetics Publishers, 1992.

LOPES AS, PIRES NETO CS. Composição corporal em militares utilizando densimetria, antropometria e impedância bioelétrica: um estudo comparativo. Revista Kinesis 1997; 17:15-30.

LUKASKI HC. Methods of the assessment of human body composition: traditional and new. American Journal of Clinical Nutrition 1987; 46:537-56.

MARTINS AL, MEMEDES MM, OLIVEIRA MPP, GUIMARÃES JNF, OLIVEIRA FP. Análise da composição corporal e do índice de massa corporal de indivíduos de 18 a 50 anos. Cad Saúde Coletiva 2001; 9(2):97-110.

NORTON K, OLDS T. Antropométrica. Rosário: Bio system, 2000

PETROSKI EL. Antropometria, técnicas e padronizações. 2ª ed. Porto Alegre: Pallotti, 2003.

PETROSKI EL, PIRES NETO CS. Análise do peso hidrostático nas posições sentada e grupada em homens e mulheres. Revista Kinesis 1992; 10:49-62.

RODRIGUEZ-AÑEZ CR, PIRES NETO CS. Desenvolvimento e validação de equações estimativas da densidade corporal de soldados e cabos do Exército Brasileiro entre 18 e 22 anos de idade. Revista Brasileira Atividade Física \& Saúde 1999; 2(4): 39-48.

SALEM M. Manual de Cineantropometria. Rio de Janeiro: Escola de Educação Física do Exército,1994.

SALEM M, AMARAL RG, CARVALHO EAM, WALZ M, NAKASHIMA GT, PUEHRINGER PH et al. Desenvolvimento e validação de equações para a estimativa da porcentagem de gordura dos alunos do curso de instrutor da Escola de Educação Física do Exército. Revista de Educação Física 2006; 133:49-58.

SALEM M, PIRES NETO CS, WAISSMANN W. Equações nacionais para a estimativa da gordura corporal de brasileiros. Revista de Educação Física 2007; 136:66-78.

SOUZA OF, PIRES NETO CS, GUIMARÃES FJSP. Comparação e validação de equações antropométricas e de impedância bioelétrica para estimar a massa corporal magra de alunos do NPOR. São Paulo: Revista Paulista de Educação Física 1998; 12(2):193-201. 
SUM SS, CHUMLEA WC, HEYMSFIELD SB, LUKASKI HC, SCHOELLER D, FRIEDL K et al. Development of bioeletrical impedance analysis prediction equations for body composition with the use of a multicomponent model for use in epidemiologic surveys. Am J Clin Nutr 2003; 77:331-40.

\section{Endereço para Correspondência:}

Marcelo Salem Instituto de Pesquisa da Capacitação Física do Exército

Av João Luís Alves, s/no - Urca

Rio de Janeiro - RJ - Brasil e-mail: marcelosalem @uol.com.br

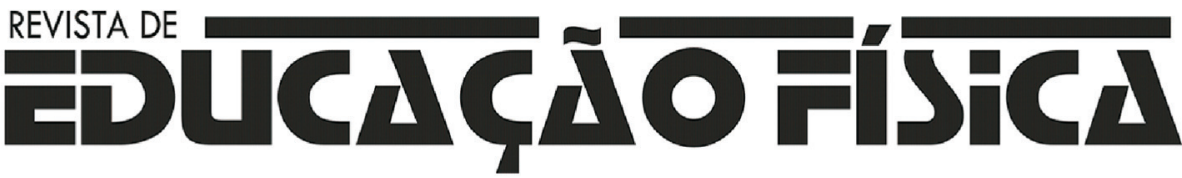

\section{A PRIMEIRA REVISTA DE EDUCAÇÃO FÍSICA DO BRASIL.}

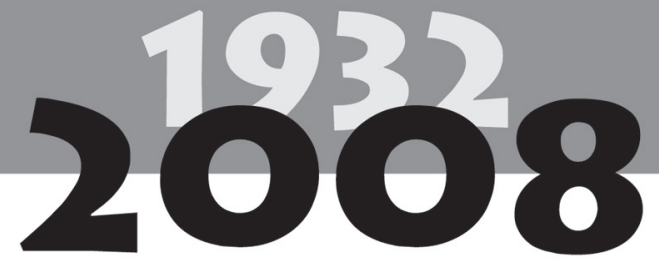

